\title{
Fortalecimiento del cuidado a cuidadores de personas con enfermedad crónica en red: resultados del estudio múlticentrico en el Norte de Santander
}

Olga Marina Vega Angarita*

Recibido:

15 de agosto de 2008

Aceptado:

3 de junio de 2009
*Enfermera Magíster en Enfermería con énfasis en cuidado al paciente crónico, Especialista Medicoquirúrgica con énfasis en neurocirugía. Docente Asociado Facultad Ciencias de la Salud, Universidad Francisco de Paula Santander, Grupo de investigación de cuidado de enfermería

E-Mail: omvega@bari.ufps.edu.co.

**Docente Asociado Facultad Ciencias de la Salud, Universidad Francisco de Paula Santander, Enfermera Magíster en Enfermería con énfasis en cuidado al paciente crónico, Especialista Docencia Universitaria. Grupo de investigación de cuidado de enfermería

E-Mail: dsgonzal@bari.ufps.edu.co.

*** Docente Asociado Facultad Ciencias de la Salud, Universidad Francisco de Paula Santander, Magíster en Enfermería con énfasis en cuidado al paciente crónico, Especialista en enfermería Nefrologica, Especialista en

Docencia Universitaria. Grupo de Investigación en Salud Publica

E-mail: mmro2000@hotmail.com

\author{
Dianne Sofía González Escobar** $\mid$ Ma. Mercedes Ramírez Ordoñez.***
}

\section{Resumen}

El proyecto realizado en el Norte de Santander hace parte del Estudio Multicentrico sobre Implementación en Red y Evaluación del Programa Cuidando a los Cuidadores, coordinado por el Grupo de Cuidado al Paciente Crónico de la Universidad Nacional de Colombia, gestora del trabajo colaborativo. Con un abordaje cuantitativo de tipo cuasi experimental, con grupos muestra y control, se realizo medición de la habilidad de cuidado a los cuidadores antes y después de la intervención y análisis comparativo de resultados. La población estuvo constituida por 50 cuidadores para ambos grupos. La investigación mantuvo los parámetros éticos para estudios con seres humanos. Se emplearon los instrumentos inventario de habilidad de cuidado ( CAI) de Ngozi O. Nkongho, caracterización de los cuidadores y el perfil Pulses.

Respecto al CAI total y el componente Valor se logró determinar que existen diferencias estadísticamente significativas entre los promedios de los grupos para los dos momentos de medición; siendo significativamente mayor en el grupo estudio tanto en la medición inicial como en la medición final $(p<0,01)$. Lo cual permite concluir que el programa fortalece la habilidad de cuidado total y el valor en los cuidadores que participan en él, y demuestra la necesidad de continuar explorando estrategias de intervención frente a la problemática de la cronicidad.

Palabras Clave: Cuidadores familiares, enfermedad crónica, programa cuidando a los cuidadores.

\section{Abstract}

He studied multicentrico on the implementation in net and evaluation of the program taking care of the caretakers, it constitutes the result of the work carried out in the North of Santander with the participation of investigators of Latin America under the coordination of the National University of Colombia agente of the collaborative work. With a quantitative boarding of quasi experimental type with groups sample and control and mensuration of the ability of care to the caretakers before and after the intervention, with comparative analysis of 
Fortalecimiento del cuidado a cuidadores de personas con enfermedad crónica en red: resultados del estudio múlticentrico en el Norte de Santander

results. The population was constituted by 50 caretakers for both groups. The investigation maintained the ethical parameters for studies with human beings. You uses the instrument inventory of ability of care of Ngozi O. Nkongho, the caretakers' instrument characterization designed by the group of care to the chronic patient of the National University of Colombia and the profile Pulses.

With regard to the I CAI total and the component Value was possible to determine that differences exist statistically significant among the averages of the groups for the two mensuration moments; being significantly bigger in the group studies so much in the initial mensuration as in the final mensuration $(p<0,01)$.

Keywords: Family carers, chronically ill, carers care program

\section{Introducción}

$\mathrm{E}^{1}$

1 mundo está experimentando un rápido aumento de los problemas crónicos de salud, hastaelpuntoquelasenfermedades crónicas constituyen actualmente más de la mitad de la carga mundial de morbilidad ${ }^{1}$.

La Organización Mundial de la salud ha documentado ampliamente el papel de las enfermedades cardiovasculares como causa principal de defunción en casi todos los países de las Américas. La mayoría de las muertes debidas a enfermedades crónicas y degenerativas se producen a una edad relativamente avanzada, como lo muestran los datos comparativos de principios de los noventa, en muchos de los países, más de la mitad de las muertes se producen a los 65 años o más tarde. Información significativa teniendo en cuenta que se prevé que en los próximos treinta años el índice de envejecimiento se duplicara o triplicara, teniendo en muchos países en el año 2025 menos jóvenes que personas mayores de 60 y mas años. ${ }^{2}$ Señala que cerca del $60 \%$ de las muertes que ocurren en el mundo hoy se deben a las enfermedades crónicas" ${ }^{3}$, en especial la enfermedad cardiaca, la enfermedad cerebrovascular, el cáncer y la enfermedad pulmonar. El informe de la OMS para 1999 advierte que estas enfermedades serán probablemente responsables del incremento en la carga de enfermedad, especialmente en los países en vías de desarrollo.

Actualmente las condiciones demográficas que caracterizan a Colombia son aumento progresivodela población, rápida urbanización, fuerte tendencia a disminuir las tasas de natalidad y aumento de la expectativa de vida al nacer. Según proyecciones del DANE entre los años 1985 y 2025 el total de la población colombiana aumentará un $168 \%$ y la población de adulto mayor crecerá $274 \%$, esta situación traerá considerables implicaciones sociales, económicas y de atención en salud. "El número de habitantes mayores de 60 años sería de 1'911.716 (6.03\% del total de la población) en 1.985, 2’900.766 (6.85\%) de la población en el 2000 y, 5’251.457 (9.87\%) de la población en el 2025"4.

A nivel regional, las enfermedades crónicas no transmisibles especialmente las cardiovasculares y cáncer se han constituido en un problema de salud publica en el Norte de Santander, "presentando una mortalidad creciente y una proporción del 17.24\% respectivamente del total ocupando el segundo lugar después de la agresión por disparo en el año de 1.998 y siendo la enfermedad cardiocerebrovascular la primera causa de muerte por enfermedades crónicas" 5

Información del Boletín Epidemiológico del año $2005^{6}$, destaca en el tercer lugar de las diez primeras causas de morbilidad por consulta externa y urgencias en todas las 
edades, la hipertensión arterial con una tasa de 33,40 por 1.000 habitantes, en el grupo de edad de 45 a 59 años; en el grupo de 60 y mas años la hipertensión arterial ocupa el segundo y primer lugar respectivamente. De otra parte, como causas de morbilidad hospitalaria se encuentra en el grupo de edad de 45 a 59 años como primeras causas la enfermedad mental no especificada y la insuficiencia cardiaca congestiva. En el grupo de edad de 60 y más años, las primeras causas de morbilidad hospitalaria están7 representadas por la Insuficiencia cardiaca congestiva, la hipertensión arterial, la enfermedad cardiaca hipertensiva y el accidente cerebro vascular.

La trascendencia de esta situación, con tendencia al aumento, reside no sólo en las tasas de morbilidad y mortalidad sino en su larga duración y elevado número de discapacitados que originan carga social, familiar y económica, y representan años de vida productiva perdidos.

Las limitaciones y discapacidades que provocan las enfermedades crónicas afectan tanto la calidad de vida del individuo como la de su cuidador o cuidadores. Ante este evento los cuidadores deben asumir una serie de tareas de cuidado que requieren del desarrollo de habilidades cognitivas y actitudinales.

Frente a esta realidad es de resaltar como importantes organizaciones en salud han definido políticas y estrategias que atiendan esta problemática. La OPS, al respecto planteo frentes estratégicos de acción de acuerdo con la estrategia mundial para la prevención y el control de las enfermedades no transmisibles. La iniciativa CARMEN (Conjunto de Acciones para la Reducción Multifactorial de las Enfermedades), que ha sido adoptada por varios países miembros, entre ellos Colombia.

A nivel del país y específicamente en el departamento Norte de Santander, se han logrado algunos avances en el diagnóstico de algunas enfermedades crónicas que lamentablemente no se han cristalizado en programas y proyectos de atención continuos e integrales. Con relación a la diabetes y la hipertensión, por ejemplo, se encuentran experiencias y registros de acciones de prevención con el propósito de disminuir la incidencia con acciones orientadas especialmente al diagnóstico precoz y captación de pacientes para intervenciones puntuales en programas que no han tenido continuidad.

Sin embargo, la situación actual de las enfermedades crónicas en el país y en la región, está acompañada de una problemática que no ha sido abordada por los servicios de salud y por enfermería, las personas que asumen la responsabilidad de cuidado en casa del enfermo crónico, los cuidadores familiares, y el impacto que este cuidado tiene en su salud física y mental, en su desarrollo personal, espiritual, familiar, social y económico, y en su calidad de vida.

En razón a que el objetivo de la atención de enfermería es ayudar a las personas a conseguir la salud, bienestar y calidad de vida óptimos que requieren, recibiendo cuidados que sean coherentes con las necesidades, los valores, deseos y contexto de la persona; el cuidado de las personas que viven en situación crónica de enfermedad requiere un abordaje total, que ayude al individuo y a su familia a adoptar medidas orientadas a reducir o eliminar de ser posible los trastornos y discapacidades a corto, mediano y largo plazo; a reducir al mínimo el sufrimiento, como también de facilitar los procesos de adaptación del paciente y la familia a las dolencias o situaciones irremediables secundarias a la enfermedad.

El Consejo Internacional de Enfermeras (CIE), como federación de las asociaciones nacionales de enfermeras de 125 países, y como portavoz internacional de la enfermería establece que los profesionales de enfermería han de desempeñar una función importante en la pronta detección e intervención, y han de participar en la promoción de la salud, prevención de la enfermedad, en la docencia y en el asesoramiento de programas para las personas discapacitadas y sus familias. 
El conocimiento que los profesionales de enfermería tengan sobre el grado de complejidad de la situación crónica, la habilidad que tiene el cuidador para ofrecer cuidado y los apoyos disponibles para el cuidado, permiten establecer prioridades de acción y formular estrategias educativas, asistenciales e investigativas, conducentes a mejorar la situación de salud en la que se encuentran inmersas estas personas.

En América Latina se ha observado que las personas que tienen un familiar con enfermedad crónica tienen la obligación de desempeñarse como cuidadores, pero no cuentan con la orientación requerida. Se ha documentado de manera importante una deficiente habilidad de cuidado entre estos cuidadores familiares, pero también de han identificado en ellos fortalezas diferentes a las de otras latitudes que pueden apoyar el servicio requerido como disponibilidad de soporte para los cuidadores?

El cuidador familiar, es la persona que presta cuidado de forma regular y cotidiana de diferentes formas; cuidado directo e instrumental en relación con el baño e higiene del paciente, alimentación, comodidad y confort, administración y control de tratamiento farmacológico y algunos procedimientos básicos necesarios para la estabilidad y bienestar de la persona enferma, sin contar con sus responsabilidades básicas de mantenimiento del hogar, trabajo, etc. Igualmente da cuidado indirecto, cuando supervisa, responde por las decisiones y da protección al paciente. ${ }^{8}$

Bajo este referente local, es válido afirmar que el desarrollo de habilidades del cuidado de los cuidadores se relaciona con factores ambientales y situacionales. Planteando la urgente necesidad de profundizar en ésta área. $\mathrm{Si}$ bien se han adelantado investigaciones sobre el cuidado de los cuidadores en la última década, la mayoría de los estudios son de carácter descriptivo y su aplicabilidad es restringida, por lo que poco apoyan a la situación real de estas situaciones ${ }^{9}$. Por otra parte llama la atención la escasa información sobre los aspectos positivos de la experiencia humana de cuidar.

En nuestros servicios de salud el cuidador es desconocido, no se le tiene en cuenta o se considera que no sabe, y no se han generado mecanismos sólidos y colectivos para apoyarlo en su responsabilidad. De forma paradójica, cada día con mayor frecuencia el cuidador recibe nuevas delegaciones para las cuales no está capacitado. Le entregamos un familiar enfermo o discapacitado porque el costo institucional de hospitalización es muy alto para las empresas prestadoras de servicios de salud, o porque no existen servicios adecuados y suficientes de cuidado a largo plazo, y el cuidador debe llevar a esta persona al hogar sin saber que sucederá.

Es entonces necesario desarrollar proyectos donde enfermería trabaje con esquemas que promuevan la salud total de quienes viven en situación crónica de enfermedad y orienten sus esfuerzos al cuidado integral, el fomento de la autonomía, el respeto y la dignidad de la persona en esta situación en su grupo familiar y social.

Para desarrollar este tipo de proyecto es indispensable, incluir al Cuidador (es) real y potencial de las personas en situación de enfermedad crónica, pues son protagonistas importantes, aunque hasta hace poco invisibles, de los procesos de atención, rehabilitación y cuidado permanente. Son personas que sin sufrir la enfermedad se enfrentan a la realidad de sus complicaciones, asumiendo un rol para el que generalmente no se encuentran preparados, modificando su calidad de vida en los aspectos: social, emocional, laboral, económico, familiar y condición de salud.

El presente estudio pretende evaluar la efectividad del programa cuidando a los cuidadores para el desarrollo de habilidades de cuidado en cuidadores familiares de personas adultas con enfermedad crónica en Cúcuta, Norte de Santander. Busca aportar al conocimiento y practica de enfermería 
a través de la experiencia de cuidado de los cuidadores familiares de personas que se encuentran en situación de enfermedad crónica. La responsabilidad de asumir el cuidado demanda al cuidador el desarrollo de habilidades cognoscitivas y actitudinales complejas para el ejercicio protagónico del cuidado en casa.

Solo a través de las experiencias de interacción y encuentro con los cuidadores de las personas en situación crónica de enfermedad, es posible construir conocimiento, alcanzar la comprensión de lo que esta experiencia representa y validar intervenciones terapéuticas que posibiliten el desarrollo de las habilidades necesarias para el ejercicio del cuidado y de su propio autocuidado, con herramientas y recursos adecuados y suficientes para enfrentar los cambios a los que el desempeño de este rol los aboca en todas sus dimensiones, con la mayor seguridad, autonomía e independencia posible.

El entrenamiento ha demostrado ser una herramienta útil, aun en los casos de mas difícil manejo como lo son la situaciones de demencia. Dentro del mismo es preciso desarrollar el conocimiento, apoyar en la adaptación del ambiente cotidiano, respaldar la toma de decisiones, ayudar a establecer y reconocer las redes de soporte social y encontrar significado en cada uno de los actos del cuidado y de las opciones para compartir una vida que puede irradiar luz suficiente para iluminar el sendero de los demás miembros de la misma familia. ${ }^{10}$

Representa una oportunidad para la cualificación del cuidado de enfermería a las personas en esta situación, la integración de la docencia y la asistencia alrededor de las necesidades de salud del contexto, el desarrollo de procesos administrativos centrados en el cuidado que respondan a las condiciones actuales de organización y prestación de servicios de salud.

Permitirá igualmente, un acercamiento y mayor comprensión de lo que representa para el cuidador la experiencia de cuidar a una persona en situación de enfermedad crónica y cambios percibidos en ella con el desarrollo de habilidades cognoscitivas y actitudinales, representadas en las tareas e intervenciones de índole instrumental y emocional que debe realizar, la adaptación a los cambios de rol y en las relaciones personales y familiares.

\section{Materiales y métodos}

Desde lo metodológico el estudio utilizó un diseño de corte cuasi experimental con abordaje cuantitativo. Los grupos a estudio (muestra y control) estuvieron conformados equitativamente por 50 cuidadores familiares, usuarios de la IPS Instituto de Seguros Sociales Francisco de Paula Santander, ESE Hospital Universitario Erasmo Meoz, Centro de Rehabilitación Cardioneuromuscular y Oncomédical; así como afiliados a grupos organizados, Asociación de Discapacitados de la Ciudadela La Libertad, que reunieron los criterios de inclusión definidos en el proyecto. El grupo muestra recibió el programa de desarrollodehabilidades decuidado"Cuidando a los Cuidadores", El grupo control participo en encuentros de apoyo convencionales. Luego de explicar los propósitos del estudio y de obtener el consentimiento informado se obtuvieron los datos del cuidador familiar y del enfermo crónico.

La información se recolectó por medio de los instrumentos Caracterización de los cuidadores, Inventario para la habilidad de cuidado ( CAI) y Escala PULSES. En la identificación de las características sociodemográficas de los cuidadores participantes se utilizó un cuestionario que identifica el sexo, edad del cuidador y del paciente, nivel de escolaridad, estado civil, ocupación, nivel socioeconómico, cuidado desde el momento del diagnóstico, tiempo de cuidado y parentesco con la persona cuidada. La determinación del nivel de funcionalidad del enfermo crónico en términos de su capacidad física y cognitiva fue medida a través del perfil "PULSES" creada por 
Moskowitz en 1957. Esta escala refleja los niveles de funcionalidad en seis parámetros: estabilidad de la patología, utilización de los miembros superiores, locomoción, capacidad sensorial, eliminación, y actividad social según lo esperado; con una graduación tipo likert; donde uno (1) equivale a independiente y cuatro (4) a completamente dependiente.

La evaluación del grado de habilidad de cuidado de una persona para cuidar a otros, se realizo con el instrumento diseñado por la doctora Ngozi O. Nkongho "Inventario para la habilidad de cuidado", denominado CAI®, Caring Hability Inventory. Instrumento que ha mostrado un grado de validez de $0.80 \mathrm{y}$ 0.84 de confiabilidad. Constituido por treinta y siete ítems (37), agrupados en las categorías conocimiento (14), valor (10) y paciencia (13). La estructuración de respuesta tipo Likert del instrumento permite su autoadministración e indican los puntajes más altos mayor grado de habilidad de cuidado para un ítem formulado positivamente; en los ítems negativamente formulados, el puntaje se invierte. Las respuestas a los diferentes ítems se suman en cada sub-escala, dando un puntaje total de habilidad y por cada subescala o categoría. La sub-escala Conocimiento se determina a través de 14 ítems, con un posible rango de puntaje de 14 a 98 . Incluye los ítems número $2,3,6,7,9,19,22,26,30,31,33,34,35$ y 36 del instrumento. La sub-escala Valor tiene 13 ítems, con posible rango de puntaje de 13 a 91, que corresponden a los ítems 4,8,11,12,1 $3,14,15,16,23,25,28,29$ y 32 del instrumento. La sub-escala Paciencia tiene 10 ítems y su puntaje puede ir de 10 hasta 70, los ítems del instrumento que corresponden a esta categoría son el 1,5,10,17,18,20,21,24,27 y 37 .

Los datos obtenidos a través de los cuestionarios iníciales y finales se incorporaron a una matriz en Microsoft Excel y se procesaron en el software estadístico MINITAB versión 14.0, con el cual se generaron estadísticas descriptivas e inferenciales.

\section{Resultados}

ISSN 0122-820X

\section{Características de los cuidadores.}

En el grupo estudio, el $82 \%$ de los cuidadores eran mujeres y el $18 \%$ hombres. El rango de edad predominante estaba en los mayores de 60 años con un 48\%, seguido de 36 a 59 años con el $42 \%$ y de 18 a 35 años en el 10\% de los casos. La mayoría se dedica al hogar $(64 \%)$ y el $26 \%$ labora como independiente. En cuanto al nivel de escolaridad se logró identificar que el $52 \%$ de los cuidadores del grupo solo ha estudiado algún grado de primaria, con un nivel de analfabetismo del 14\%. El 80\% de los cuidadores tenían más de 37 meses en el desempeño del rol, el $10 \%$ entre 0 y 6 meses, el $6 \%$ de 7 a 18 meses y el 4\%. En el 68\% de los casos la persona es el único cuidador del enfermo crónico, dedicando a las acciones de cuidado las 24 horas del día (66\%). Respecto a la relación o parentesco con la persona cuidada, el $40 \%$ de los cuidadores son padre ó madre del paciente, $24 \%$ esposa(o) del paciente y el $22 \%$ son hijas(os). El $48 \%$ de los pacientes se encuentra en un rango de edad mayor al de su cuidador, mientras que el $46 \%$ se encuentra en un rango inferior. Sólo el 6\% de los pacientes se encuentra en el mismo rango de edad del cuidador. Respecto al nivel de funcionalidad del enfermo crónico medido con la escala PULSES, el $48 \%$ de los receptores de cuidado tienen un compromiso severo, el 30\% un compromiso moderado y el $22 \%$ un compromiso leve.

En el grupo control, el $84 \%$ de los cuidadores eran mujeres y el $16 \%$ hombres. La edad del cuidador estaba entre 36 a 59 años en un $52 \%$ de los casos, entre 18 a 35 años en el 34\% y un $10 \%$ eran mayores de 60 años. La ocupación de los cuidadores de este grupo es principalmente el hogar en un $54 \%$, el $26 \%$ labora de manera independiente y el $16 \%$ es empleado. En cuanto al nivel de escolaridad, el $56 \%$ de los cuidadores han realizado estudios de secundaria, con un 
nivel de analfabetismo del 2\%. El $42 \%$ de los cuidadores lleva un periodo inferior a 6 meses en la experiencia de cuidar, el $26 \%$ más de 37 meses y el $24 \%$ entre 7 y 18 meses. Dedican a la labor de cuidar al familiar enfermo las 24 horas del día el $44 \%$ de los casos, entre 7 y 12 horas el $22 \%$, entre 13 y 23 horas el $12 \%$ y menos de 6 horas al día el 22\%. Un $38 \%$ de los cuidadores no cuentan con apoyo en la labor de cuidado del enfermo crónico. En cuanto a la relación de parentesco, el 30\% de los cuidadores son el padre ó la madre del paciente, el $20 \%$ son el esposo ó la esposa del paciente y el 24\% son hijos (as) del paciente. El $48 \%$ de los pacientes se encuentra en un rango de edad mayor al de sus cuidadores, el $46 \%$ se encuentra en un rango de edad inferior y el $6 \%$ de los pacientes se encuentra dentro del mismo rango de edad que la de su cuidador. El compromiso del nivel de funcionalidad de los enfermos crónicos de acuerdo a la escala PULSES reportó un compromiso severo en el $52 \%$ de los casos, compromiso moderado en el $30 \%$ y con compromiso leve un $18 \%$ de los pacientes.

\section{Habilidad de cuidado de los Cuidadores.}

Figura 1. Clasificación general habilidad de cuidado inicial se ubico en 205.6 pasando a 206.5 en la valoración final; mientras en el grupo control, la valoración inicial registro un promedio de 195.6 pasando a 199.9 en la valoración final. El valor promedio de habilidad de cuidado total a nivel general presento un incremento en la valoración final respecto a la valoración inicial, tanto en el grupo estudio como en el grupo control. (Figura 1.).

En el componente conocimiento se presentó un incremento en el valor promedio en la medición final en relación con la medición inicial en ambos grupos, significativamente en el grupo control. Para el grupo estudio se encontró que el promedio pasó de 82.8 (DS 10.8) a 83.0 (DS 8.3); mientras que en el grupo control el valor promedio pasó de 81.2 (DS 11.6) en la medición inicial a 89.2 (DS 8.4) en la medición final.

El componente paciencia para el grupo estudio presento una mediana de 62.5 en la medición inicial (DS 8.4) y una mediana de 61 (DS 7.3) en la medición final. Por su parte el grupo control presento una mediana de 62 en la medición inicial (DS 8.1) pasando a una mediana de 67 y (DS 4.6) en la medición final.

El componente valor para el grupo estudio presento una mediana de 60 en la medición inicial (DS 13.4) y una mediana de 63.5 (DS 13.2) en la medición final. Por su parte el grupo control presento una mediana de 56 en la medición inicial (DS 12.4), pasando a una mediana de 44 (DS 10.5) en la medición final.

\section{Comparativo por grupos.}

El valor promedio para habilidades de cuidado a nivel general presentó unincremento en su valoración final con respecto a la valoración inicial, tanto en el grupo estudio como en el grupo control (Figura 2.).

Con respecto al componente conocimiento, igualmente se presentó un incremento en el valor promedio en la medición final en relación analizada de manera total y por componentes, antes y después de la intervención, en el grupo estudio y control. El valor promedio del CAI Total se ubico en 200.6 en la valoración inicial, pasando a 203.2 en la valoración final. En el grupo estudio, el valor promedio 
Fortalecimiento del cuidado a cuidadores de personas con enfermedad crónica en red: resultados del estudio múlticentrico en el Norte de Santander

con la medición inicial, significativamente en el grupo control; Para el grupo estudio, el valor promedio pasó de 82,8 a 83 , mientras que en el grupo control el valor promedio pasó de 81,2 en la medición inicial a 89,2 en la medición final (Figura 3).

Figura 2. Comparativo general para resultados de habilidad de cuidado

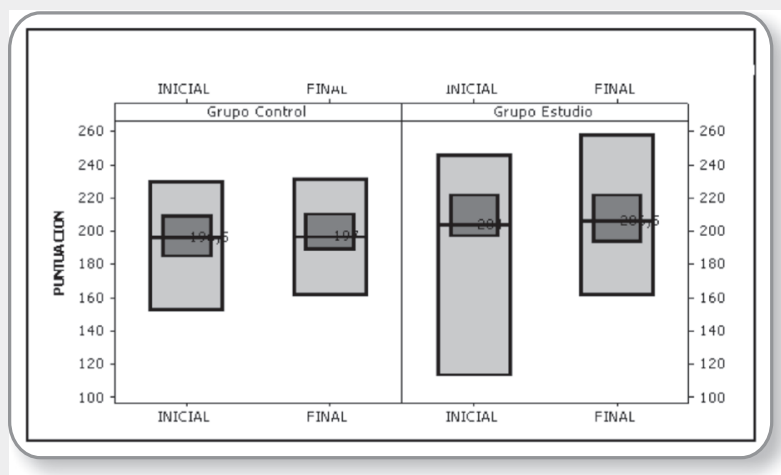

Figura 3. Comparativo resultados componente conocimiento

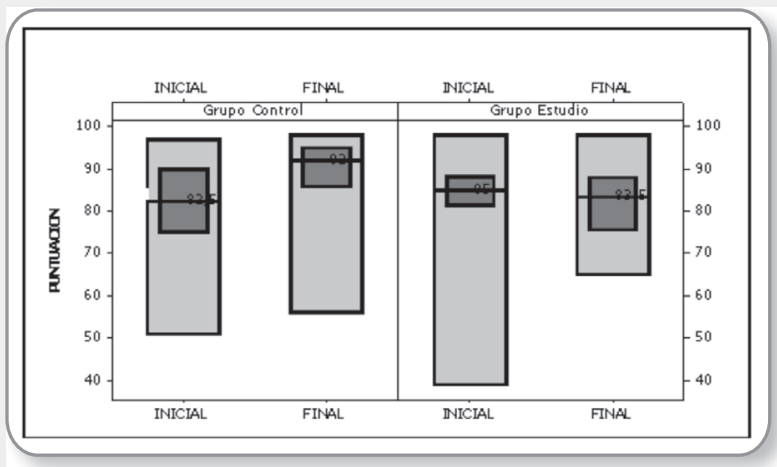

En el componente paciencia a pesar de que a nivel general se presentó un leve incremento entre una medición y otra, se logró determinar que en el grupo estudio presentó una disminución del valor promedio en su medición final, ya que pasó de 60,8 en la valoración inicial a 59,8 en la valoración final. En el grupo control por el contrario, se observó un incremento significativo, puesto que pasó de 60,5 en la valoración inicial a 64,8 en la medición final (Figura 4.)

Caso contrario se registró con respecto al componente valor, donde se logró observar un leve incremento del valor promedio en el grupo estudio, pasando de 62 en la medición inicial a 63,7 en la medición final. En cambio se registró una disminución bastante significativa en el valor promedio en el grupo control, ya que paso de 53,9 a 45,9 en su medición final, lo cual repercutió considerablemente en el promedio general (Figura 5).

Figura 4. Comparativo resultados componente paciencia

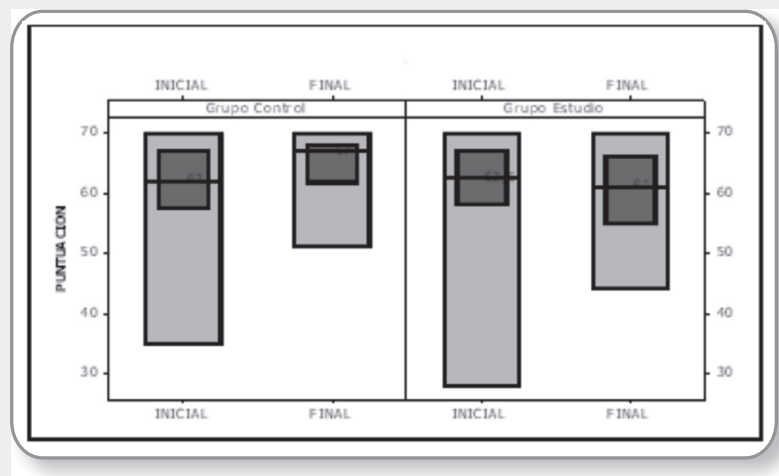

Figura 5. Comparativo resultados componente valor

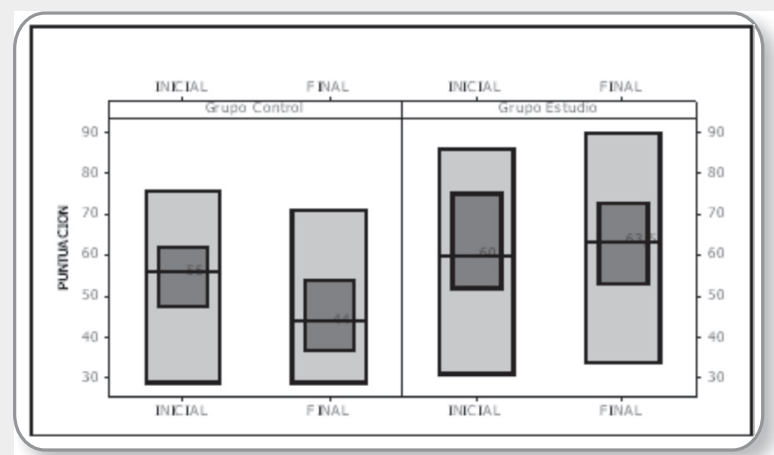

\section{Análisis comparativo}

Los grupos estudio y control fueron comparados en cada momento de medición, empleando la prueba t de Student, con un nivel de significancia de 0,05 , para lo cual se contrasto la hipótesis de diferencia entre las medias de los grupos.

ConrespectoalCAItotal,selogródeterminar que existen diferencias estadísticamente significativas entre los promedios de los grupos para los dos momentos de medición; es decir, el valor promedio para habilidades de cuidado es significativamente mayor en 
el grupo estudio tanto en la medición inicial como en la medición final $(\mathrm{p}<0,01)$.

Para el componente conocimiento, se logró determinar que en su valoración inicial, el valor promedio del grupo estudio es mayor que en el grupo control; pero en la valoración final, el valor promedio es significativamente mayor en el grupo control que en el grupo estudio. Caso similar ocurre en el componente paciencia, donde no existen diferencias en su valoración inicial, pero en la valoración final, el valor promedio registrado en el grupo control es mayor al registrado en el grupo estudio.

Con respecto al componente valor se logró determinar diferencias estadísticamente significativas entre los promedios grupales; es decir, el valor promedio registrado en el grupo estudio tanto en su valoración inicial como en la medición final, es significativamente mayor al promedio registrado en el grupo control $(\mathrm{p}<0,01)$ (Tabla 1).

Tabla 1. Comparación entre los grupos estudio y control, antes y despues de la intervención

\begin{tabular}{|l|c|c|c|}
\hline \multirow{2}{*}{ Categoría } & \multicolumn{2}{|c|}{ Momento de medición } & \multirow{2}{*}{ t de referencia } \\
\cline { 2 - 3 } & Inicial & Final & \\
\cline { 2 - 3 } & Valor de t & Valor de t & \\
\hline CAI Total & 10,79 & 7,87 & $\pm 2,0141$ \\
\hline Conocimiento & 2,38 & $-10,59$ & $\pm 2,0141$ \\
\hline Paciencia & 0,42 & $-10,26$ & $\pm 2,0141$ \\
\hline Valor & 11,31 & 25,87 & $\pm 2,0141$ \\
\hline
\end{tabular}

Al realizar la comparación de los momentos de medición del CAI total y los componentes en cada uno de los grupos, se determinó que a nivel general no se encontraron diferencias significativas entre el valor promedio inicial y final en el grupo estudio, observándose únicamente un incremento significativo en cuanto al componente valor $(\mathrm{p}<0,05)$. En el grupo control, tanto a nivel general como en el componente conocimiento y el componente paciencia se registró un incremento significativo en la medición final, mientras que en el componente valor se presentó una disminución significativa en su medición final $(\mathrm{p}<0,05)$ (Tabla 2).
Tabla 2. Comparación de los momentos de medición para cada uno de los grupos

\begin{tabular}{|l|c|c|c|}
\hline \multirow{2}{*}{ Categoría } & \multicolumn{2}{|c|}{ Grupo } & \multirow{2}{*}{ Control } \\
\cline { 2 - 3 } & Estudio & Ce referencia \\
\cline { 2 - 3 } & Valor de t & Valor de t & \\
\hline CAI Total & 0,95 & 5,33 & $\pm 2,0141$ \\
\hline Conocimiento & 0,35 & 12,56 & $\pm 2,0141$ \\
\hline Paciencia & $-1,71$ & 8,51 & $\pm 2,0141$ \\
\hline Valor & 2,27 & $-11,84$ & $\pm 2,0141$ \\
\hline
\end{tabular}

\section{Discusión}

Se encontró similar distribución por género en ambos grupos vinculados a la investigación. Los resultados obtenidos son apoyados por la literatura de enfermería en la que establecen que la preocupación por la salud y el cuidado del enfermo en el hogar ha sido por mucho tiempo responsabilidad de un familiar, generalmente una mujer (madre, hija, esposa, abuela) ${ }^{11}$

Se reporta en ambos casos mayor porcentaje de cuidadores familiares en el rango de 36 a 59 años, etapa en la que los cuidadores se encuentran en su periodo de mayor productividad y desarrollo. El problema de la simultaneidad de las obligaciones y responsabilidades de cuidado es un factor de desestabilización emocional, aumento de tensiones y preocupación en el cuidador ${ }^{12}$. Es significativo para el grupo estudio, el alto porcentaje de personas mayores de 60 años que tienen la responsabilidad de cuidado de una persona con alto grado de dependencia. Situación que se hace critica en el caso de las mujeres que envejecen, muchas estarán atendiendo a sus esposos, ayudando a hijos y nietos, contando con menos apoyo familiar que les ayude a ocuparse de sus padres enfermos ${ }^{13}$

En cuanto a la escolaridad, los resultados obtenidos ubican a los cuidadores en un nivel bajo, destacándose lo encontrado en el grupo estudio. La relación con los bajos niveles de escolaridad que maneja el grupo a estudio los condiciona a ejercer actividades como labores del hogar y trabajos informales que no garantizan la estabilidad económica que ellos necesitan. El nivel de escolaridad 
puede ser una característica importante en los cuidadores, al ampliar las posibilidades de acceso a la información y desarrollo de procesos de aprendizaje por diferentes medios de comunicación acerca del cuidado, el proceso de la enfermedad y de los mecanismos para mejorar el bienestar de la persona receptora de cuidado.

La distribución de acuerdo al estado civil es muy similar en ambos grupos vinculados a la investigación. Esta característica puede relacionarse con la edad de los cuidadores, la relación y vinculo con la persona cuidada de madre/padre, esposa(o) e hija(o). Información significativa, si se tiene en cuenta que estos cuidadores deben desempeñar múltiples roles además del de cuidador, así como las situaciones que la responsabilidad de cuidar a una persona crónicamente enferma puede generar en la dinámica familiar, la vida de pareja y otros aspectos como el económico y social.

La mayoría de los cuidadores se dedican al hogar, siendo esta ocupación de mayor prevalencia en el grupo estudio. Lo encontrado en el estudio explica en alguna medida la condición socioeconómica encontrada los cuidadores participantes, estratos 1 y 2; así mismo guarda correspondencia con las características sexo y nivel educativo anteriormente descritas, al ser el cuidado del miembro de la familia enfermo una de las funciones del rol que desempeña la mujer en el hogar; por su parte los desempleados y trabajadores independientes pueden tener mayor disponibilidad de tiempo para el desempeño de las tareas derivadas del cuidado dentro del grupo familiar. Según Francisco del Rey y col. (1995), las amas de casa y los desempleados por disponer del tiempo requerido, son los cuidadores más frecuentes de los enfermos crónicos.

En ambos grupos poco más de la mitad de los cuidadores llevan más de 36 meses en rol de cuidador, seguido de 18 meses ó menos. En el grupo estudio es mayor el tiempo de experiencia como cuidadores, dedicando todo el día a esta labor. Lo encontrado en el estudio implica la dedicación considerable de tiempo y energía en la atención de complejas actividades no siempre cómodas y agradables, ni previstas a su libre elección ${ }^{14}$, una experiencia ingrata y solitaria que termina por reducir los niveles de paciencia y del conocimiento de sí mismo. Es significativo el porcentaje de cuidadores del grupo estudio que se desempeñan como únicos cuidadores, la literatura refiere que la cantidad y tipo de ayuda varía en función del grado y de la causa que haya determinado dependencia de la persona cuidada. Cuanto mayor sea el grado de dependencia, mayor será también la cantidad de tiempo y de esfuerzo que tendrá que dedicar el cuidador "inexperto" a la tarea de cuidar a su familiar.

En cuanto a la relación con la persona cuidada se encontró un comportamiento relativamente similar en ambos grupos, siendo las madres/ padres, hijas(os) y esposas(os) quienes asumen la responsabilidad de cuidado de un familiar crónicamente enfermo. Según Barrera L, (2002), el parentesco es un factor muy importante para comprender y entender los sentimientos y experiencias que viven los cuidadores primarios.

El comportamiento respecto a la edad del paciente es idéntico en ambos grupos vinculados a la investigación. Dada la estrecha relación de estas enfermedades con el envejecimiento, puede concluirse que el número de cuidadores jóvenes con parentescos de hijas(os) seguirá aumentando en los próximos años. Asumiendo la responsabilidad de cuidar personas con compromiso severo y moderado en su capacidad funcional, con limitación para la satisfacción de sus necesidades básicas y realización de las actividades de la vida diaria.

Respecto a la habilidad de cuidado se observan algunas diferencias en la valoración del CAI total entre los grupos antes de la realización de las intervenciones, encontrando mayores porcentajes de cuidadores con niveles entre medio y bajo de habilidad en el grupo estudio que en el grupo control. 
Similar comportamiento se observa en las categorías conocimiento y paciencia, e igualdad en el componente valor.

Después de realizada la intervención se observa un aumento en la valoración general del CAI total, determinándose que existen diferencias estadísticamente significativas entre los promedios de los grupos para los dos momentos de medición; es decir, el valor promedio para habilidades de cuidado es significativamente mayor en el grupo estudio tanto en la medición inicial como en la medición final $(\mathrm{p}<0,01)$. Lo cual guarda correspondencia con lo reportado por estudios similares ${ }^{15}$ en los cuales la habilidad total incremento significativamente después de la intervención de enfermería, particularmente en el grupo estudio con el cual se desarrolla el programa cuidando al cuidador.

En la valoración de los componentes de la habilidad de cuidado se encuentran diferencias significativas entre los grupos. En el grupo estudio el conocimiento y la paciencia se comportan de manera homogénea en las dos mediciones, por el contrario se observa un incremento representativo en el componente valor. Por su parte el grupo control muestra un incremento en los niveles del conocimiento y la paciencia, y disminución en el componente valor. En el análisis comparativo se logró determinar diferencias estadísticamente significativas entre los promedios grupales de la categoría valor; es decir, el valor promedio registrado en el grupo estudio tanto en su valoración inicial como en la medición final, es significativamente mayor al promedio registrado en el grupo control $(\mathrm{p}<0,01)$.

Al mirar la medición final de la habilidad general y la paciencia se encontró que en el grupo de estudio disminuyo en relación con el grupo control esta situación puede haberse dado porque al realizar los talleres de esta dimensión las personas realizaron una reflexión de su propio yo, teniendo más claridad de sus debilidades; al aplicar nuevamente el instrumento lo diligenciaron con una mirada más clara con relación a su propia manera de ser y actuar; donde el tiempo de medición pudo no permitir la percepción de los cambios que él podía estar efectuando en su cotidianidad. Estos resultados permiten observar que aquellos aspectos del ser humano relacionados con la actitud, que buscan producir sensibilización y cambios en la persona en su forma de actuar, necesitan espacios más prolongados y un proceso de acompañamiento que permita la expresión de dudas, temores e inseguridades con el ser al que cuidan.

En cuanto a los resultados obtenidos en la categoría conocimiento, es de considerar que el nivel de escolaridad es una característica importante en los cuidadores al ampliar las posibilidades de acceso a la información y desarrollo de procesos de aprendizaje por diferentes medios de comunicación acerca del cuidado, el proceso de la enfermedad y de los mecanismos para mejorar el bienestar de la persona receptora de cuidado. El cuidado en casa requiere que los cuidadores tengan destrezas y conocimientos cada vez más complejos $^{16}$. La importancia del conocimiento en el cuidado del enfermo lo manifiesta Fitzgerald (2000) ${ }^{17}$ al afirmar "el conocimiento se considera un proveedor de estructura que disminuye en los cuidadores la incertidumbre y permite el desarrollo de un mapa cognitivo para la continua interpretación de los eventos y toma de decisiones en la acción de cuidado".

Hallazgos descritos por otros estudios ${ }^{18,19}$ indican que más allá del tipo, la intensidad y el tiempo que tenga el cuidador ejerciendo su rol, es importante garantizarle un conocimiento adecuado, un respaldo suficiente en términos de soporte social, idoneidad en la toma de decisiones y un redimensionamiento de la experiencia misma. Los cuidadores son personas que necesitan asesoría y apoyo en cada uno de los procesos que vive, para poder desenvolverse con mayor efectividad y seguridad en la relación de cuidar al otro, con la suficiente disposición para enfrentar cada una de las etapas que se presentan.

Con relación al componente paciencia en el grupo control una de las situaciones que 
alteran la paciencia es el desconocimiento de los cuidadores en la realización de las actividades instrumentales, en primera instancia por ser una situación nueva que generalmente ocurre de forma imprevista causando en ellos niveles de estrés por sentir la falta de ayuda y de acompañamiento en este proceso, situación que en el momento de ser atendida les permite sentir más tranquilidad y persistencia para el cuidado.

El nivel de paciencia encontrado muestra como se hace necesario seguir reforzando la habilidad en esta dimensión entre los aspectos que más impacientan a los cuidadores es darse cuenta de cómo al ser que cuidan en algunas situaciones no atienden sus sugerencias, quieren imponer sus propios criterios, prestan poca atención a lo que ellos les expresan, la demanda de tiempo en el cuidado que ocasiona en algunos casos de acuerdo a las circunstancias niveles de estrés, demora para ser atendidos cuando buscan los servicios de salud, no tener la suficiente información sobre dónde acudir; existe un reconocimiento de estos aspectos pero se les dificulta ser menos impulsivos y actuar en algunas situaciones con mayor serenidad, algunos encuentran calma a través de la música, la lectura o decidir no escuchar y cambiar de lugar. Es importante seguir trabajando con este grupo de personas en aprender a escuchar al otro, de dejar opinar y llegar a puntos de encuentro, fortalecer formas de relajación que les permita actuar de forma más tranquila y serena en los momentos críticos.

El valor es un elemento que está presente en el cuidado cuando la dirección del crecimiento y su resultado es desconocido, se gana valor para cuidar de las experiencias del pasado, siendo sensible y abriéndose a las necesidades del presente. ${ }^{20} \mathrm{El}$ cuidador familiar enfrenta múltiples momentos críticos en el ejercicio de su rol, que representan oportunidades para el desarrollo y descubrimiento de esta dimensión de la habilidad de cuidado o por el contrario se constituyen en experiencias que contribuyen a la frustración y la angustia. Refiere la literatura que se han categorizado cuatro grandes aspectos relacionados con la angustia que sufre la familia de un enfermo crónico: la condición del ser querido, la atención que recibe por parte del equipo de salud, el cuidado futuro y los aspectos financieros. ${ }^{21}$

El valor es un componente de la habilidad de cuidado en la que se encuentran niveles deficientes al realizar las mediciones, seguramente por el impacto emocional permanente que la experiencia del cuidado les genera, independientemente del parentesco con el receptor de cuidado, el tiempo en el ejercicio del rol y nivel de escolaridad. El programa cuidando al cuidador fortalece esta dimensión de la habilidad de cuidado, como lo demuestran los resultados obtenidos, con diferencias estadísticamente significativas en comparación con intervenciones educativas de apoyo tradicionales; seguramente por la oportunidad de reflexión, expresión, asociación, reconocimiento y autovaloración que representa.

Al comparar los resultados obtenidos en esta dimensión con lo reportado en la evaluación de un programa para fortalecer a los cuidadores familiares de enfermos crónicos $(2006)^{22}$, se encuentra correspondencia en los resultados obtenidos al presentar un aumento significativo en el nivel de valor especialmente en el grupo estudio. El aumento de los niveles del valor en el grupo estudio, puede explicarse por las características del programa cuidando al cuidador desarrollado con ellos, que busca que el cuidador descubra su experiencia de cuidado y la analice, identifique potencialidades y limitaciones, identifique las ganancias que el proceso de ser cuidador le genera y que se empodere dentro de su rol . En los talleres de valor, las temáticas resaltan el coraje, el proceso de toma de decisiones de cuidado y la habilidad social, soporte social y empoderamiento del cuidador ${ }^{23}$. Es decir, los talleres de valor buscan que los cuidadores al compartir y revivir sus experiencias reconozcan su fortaleza y fuerza interior, admitan sus logros, crean en su capacidad y habilidad, se respeten y valoren a sí mismos y a la gran labor que realizan día a día. 
En la interacción con los cuidadores se perciben cambios de actitud frente a su quehacer como cuidador, sin embargo al analizar los resultados obtenidos en las mediciones del CAI, pareciera que éste no es sensible a estas situaciones, por su estructura, forma de redacción o capacidad de interpretación del instrumento por parte de los cuidadores participantes por su nivel educativo.

Los resultados obtenidos en el estudio dan una evaluación positiva de la efectividad del programa en el fortalecimiento de la habilidad de cuidado de las personas que cuidan a enfermos crónicos, específicamente a nivel de habilidad total o general y del valor, frente a las actividades de cuidado convencionales desarrolladas por los profesionales. Aporte significativo desde la disciplina de enfermería a la compleja situación que representan las enfermedades crónicas para las personas que las padecen y sus cuidadores familiares; y evidencia de la necesidad de continuar desarrollando estrategias de intervención conceptual y metodológicamente soportadas.

\section{Bibliografía}

1. WORLDHEALTHORGANIZATION Wordl Health Report 2004, Changing history.Geneva, 2004.

2. O R G A N I Z A C I Ò N PANAMERICANA DE LA SALUD. ORGANIZACION MUNDIAL DE LA SALUD. Envejecimiento en las Americas proyecciones para el siglo XXI. www.census.gov/ipc/prod/ageams. pdf.

3. $\mathrm{O} R \mathrm{R}$ A N I Z A C I Ò N PANAMERICANA DE LA SALUD. Washington: OPS-OMS, 1998.

4. COLOMBIA. MINISTERIO DE SALUD. Situación de salud en
Colombia, indicadores bàsicos. Bogotá: MINSALUD, 2001.

5. DEPARTAMENTO NORTE DE SANTANDER. Diagnostico de salud. San José de Cúcuta: La Gobernación, 1998. p. 15.

6. DEPARTAMENTO NORTE DE SANTANDER. INSTITUTO DEPARTAMENTAL DE SALUD. Boletin epidemiológico Norte de Santander. Vol 6. (2005). p. 18-19.

7. PINTO, Natividad, BARRERA, Lucy, SANCHEZ Beatriz, Reflexiones sobre el cuidado a partir del programa Cuidando a los Cuidadores. En: Revista Aquichan, Vol. 5 N 1 (5) Facultad de Enfermería, Chia: Universidad de la Sabana, 2005. p. 130.

8. SÁNCHEZ HERRERA, Beatriz. Habilidad del cuidado de los cuidadores de personas en situación de enfermedad crónica. el arte y la ciencia del cuidado. Facultad de Enfermería. Universidad Nacional de Colombia. Bogota: Unibiblos, 2002. p. 373.

9. PINTO AFANADOR, Natividad y SANCHEZ, Beatriz. El Reto de los cuidadores, familias de personas en situación crónica de enfermedad. Bogotá: Universidad Nacional, 2003. p. 5.

10. PINTO AFANADOR, Natividad y SANCHEZ, Beatriz. OP. Cit, p. 65.

11. LANGE Ilta, URRUTIA Mila, CAMPOS Delicia, GALLEGOS Esther, HERRERA Luz Maria, LUARTE Marcela, MANDIGAN Elizabeth. Fortalecimiento del autocuidado como estrategiadela tención primariaenSalud: La contribución de las instituciones de salud en América Latina. Organización Panamericana de la Salud. Enero 2006, p. 15 
12. QUERO Rufián Aurora, Los cuidadores en el Hospital Ruiz de Alda en Granada, [Tesis Doctoral], Universidad de Granada España, 2007.P 52. En: http:// hera.ugr.es/tesisugr/16683134.pdfhttp:// hera.ugr.es/tesisugr/16683134.pdf.

13. ANZOLA P Elías, GALINSKY David, MORALES M, Fernando, Op Cite p.366

14. MARCO Navarro Esther, Calidad de vida del cuidador del hemipléjico vascular, Facultad de Medicina Universitat Autonoma de Barcelona, España 2004, Op.Cite. P. 61

15. BARRERA ORTIZ, LUCY, PINTO AFANADOR NATIVIDAD Y SANCHEZ HERRERA BEATRIZ. Evaluación de un Programa para fortalecer a los Cuidadores Familiares de Enfermos Crónicos. REVISTA SALUD PUBLICA. VOL.8 (2), JULIO 2006. Pag 141 - 152 pag 148

16. SCHUMCHER KL, STEWART BJ, ARCHBOLD PG, DODD MJ, DIBBLE SL. Family caregving skill: development of the concept. Res Nurs Health. 2000; 23(3): 191-203.

17. FITZGERALD MILLER, Judith. Recursos de Poder del Cliente. Philadelphia. Davis, 2000. P.3-16

18. BARRERA ORTIZ, Lucy, BLANCO DE CAMARGO, Lidia, FIGUEROA Ingrid, PINTO AFANADOR, Natividad, SÁNCHEZ HERRERA, Beatriz La Habilidad de Cuidado de los Cuidadores Familiares de personas con Enfermedad Crónica: Un Estudio Comparativo en tres Países de América Latina. Documento Grupo de Cuidado al Paciente Crónico. Facultad de Enfermería. Universidad Nacional de Colombia. Bogotá. 2.004.p. 8 - 9
19. BARRERA ORTIZ, Lucy, BLANCO DE CAMARGO, Lidia, FIGUEROA Ingrid, PINTO AFANADOR, Natividad, SÁNCHEZ HERRERA, Beatriz. Habilidad de cuidadores familiares de personas con enfermedad crónica. Mirada internacional, Revista Aquichan. Universidad de la Sabana, Bogotá; Vol. 6, No. 1 (2006); p. 22-33.

20. NGOZI O. NKONGHO. The Caring Ability Inventory. En: Strickland the O. Waltz, C. Measurement of Nursing Outecomes. Volumen FORU.1.999

21. PINTO A, Natividad, BARRERA O, Lucy, SANCHEZ HERRERA, Beatriz. Reflexiones sobre el cuidado a partir del Programa "cuidado a los Cuidadores". En revista: AQUICHAN.Año 5- Vol 5.№ 1(5) 2005.ISSN 1657-5997. P 128 137

22. SANCHEZ HERRERA, Beatriz, BARRERA ORTIZ, Lucy, PINTO AFANADOR, Natividad. Programa " Cuidando a los Cuidadores". COLCIENCIAS. Universidad Nacional de Colombia. 2004 . p.1 